\title{
Mechanisms of Neural Blockade
}

\author{
Andreas Grabinsky, MD
}

Background: Neural blockade remains a key diagnostic and treatment modality for interventional pain management. Most of the techniques used in chronic and acute pain management today evolved out of different techniques used by surgeons and anesthesiologists alike to facilitate surgical procedures and alleviate intra- and postoperative pain. Many of these techniques were de- veloped and introduced into medical practice during the late $19^{\text {th }}$ and early $20^{\text {th }}$ centuries and were only recently challenged by increasing neurophysiologic understanding and modern imaging studies. A brief historical overview of the topic of neural blockade as well as current knowledge of mechanisms and clinical aspects of nerve blocks are considered. The review focuses on relevant as- pects of anatomy, cell physiology, molecular mechanisms of effect, and clinical aspects of nerve blocks.

Keywords: Neural blockade, peripheral nerve blocks, neuro-axial nerve blocks, nerve impulse generation and conduction, regional anesthesia, pain management procedures.
Originally, regional anesthesia was performed by compressing peripheral nerves over an extended period of time to cause profound long lasting anesthesia, distal to the site of compression. This could also produce long lasting nerve damage as well. This method of nerve compression was described in the $16^{\text {th }}$ century by the French military surgeon Ambroise Pare (1510-1590), but the technique may have been used earlier but not reported. Another famous French military surgeon, Dominique Jean Larrey (17661842), who served under Napoleon as the surgeon-in-chief of the French army, described his observations of cold injuries on nerve function and their effect on reducing the pain of amputation.

While the anesthetic properties of cocaine were known and published

From: Department of Anesthesiology, Metro Health Medical Center and Case Western Reserve University School of Medicine, Cleveland, Ohio Address Correspondence:

Andreas Grabinsky, MD

Assistant Professor of Anesthesiology

Metro Health Medical Center and

Case Western Reserve University School of

Medicine

Cleveland, $\mathrm{OH} 44106$

E-mail: grabi@mac.com

Disclaimer: There was no external funding in the preparation of this manuscript.

Conflict of Interest: None

Manuscript received on $7 / 28 / 2005$

Revision submitted on $8 / 9 / 2005$

Accepted for publication 9/1/2005 in the $19^{\text {th }}$ century, it was Carl Koller (1852-1944), an Austrian physician, who recognized the importance of those findings and introduced cocaine into clinical practice as a local anesthetic. In 1884 , Koller prepared an aqueous solution of cocaine and instilled the solution onto the cornea to anesthetize the eye of a frog. The results of the study were presented at the German Ophthalmological Society meeting in Heidelberg later that year (1).

Today, regional anesthesia is performed most frequently by injection of local anesthetic into close proximity of specific target sites in the central and peripheral nervous system causing a temporary neural blockade, depending on the pharmacologic properties of the injected local anesthetic. However, other forms of regional anesthesia include radiofrequency ablation, chemical denervation, heat lesions, cold lesions and surgical interruption of nervous pathways. These other forms of regional anesthesia are more permanent in nature than are local anesthetics; they have higher complication rates, and are not suited as a technique for regional anesthesia for surgical procedures. However, they may have use as treatment for chronic pain states, where the benefits of long duration outweighs the risks and side effects of the destructive procedure. The idea behind these neuro-destructive procedures was to allow permanent effect anesthesia compared to the temporary effect of local anesthetics. But because of nerve plasticity and the re-growth of nerve axons after peripheral damage and Wallerian degeneration, these procedures offer analgesia up to several months.

Regional anesthesia is performed at various levels of the nervous system, using local aesthetics administered topically, by tissue infiltration, intravenous injections, peripheral nerve blocks and neuroaxial blocks. Most of these blocks prevent the generation or propagation of sensory afferent impulses in the axon of the first neuron, whose cell body is localized in the dorsal root ganglion. Central neuroaxial blockade, including spinal and epidural anesthesia, affects spinal nerves or interrupts signal transmission in the dorsal roots or dorsal root ganglia of the spinal cord (2). Besides inhibiting afferent impulses, regional anesthesia also affects motor function by blockade of efferent motor and autonomic nerve fibers.

The endpoint for all these approaches to regional anesthesia is the same. A local anesthetic needs to reach voltage-gated sodium channels on the nerve cell in order to block the conduction of nerve impulses by inhibiting propagation of the action potential. If the blockade is sufficient in magnitude to block nerve transmission associated with painful surgical stimuli, the result will be a satisfactory regional anesthetic.

Depending on the kind of regional anesthetic performed and the site of injection utilized, the onset, duration, vol- 


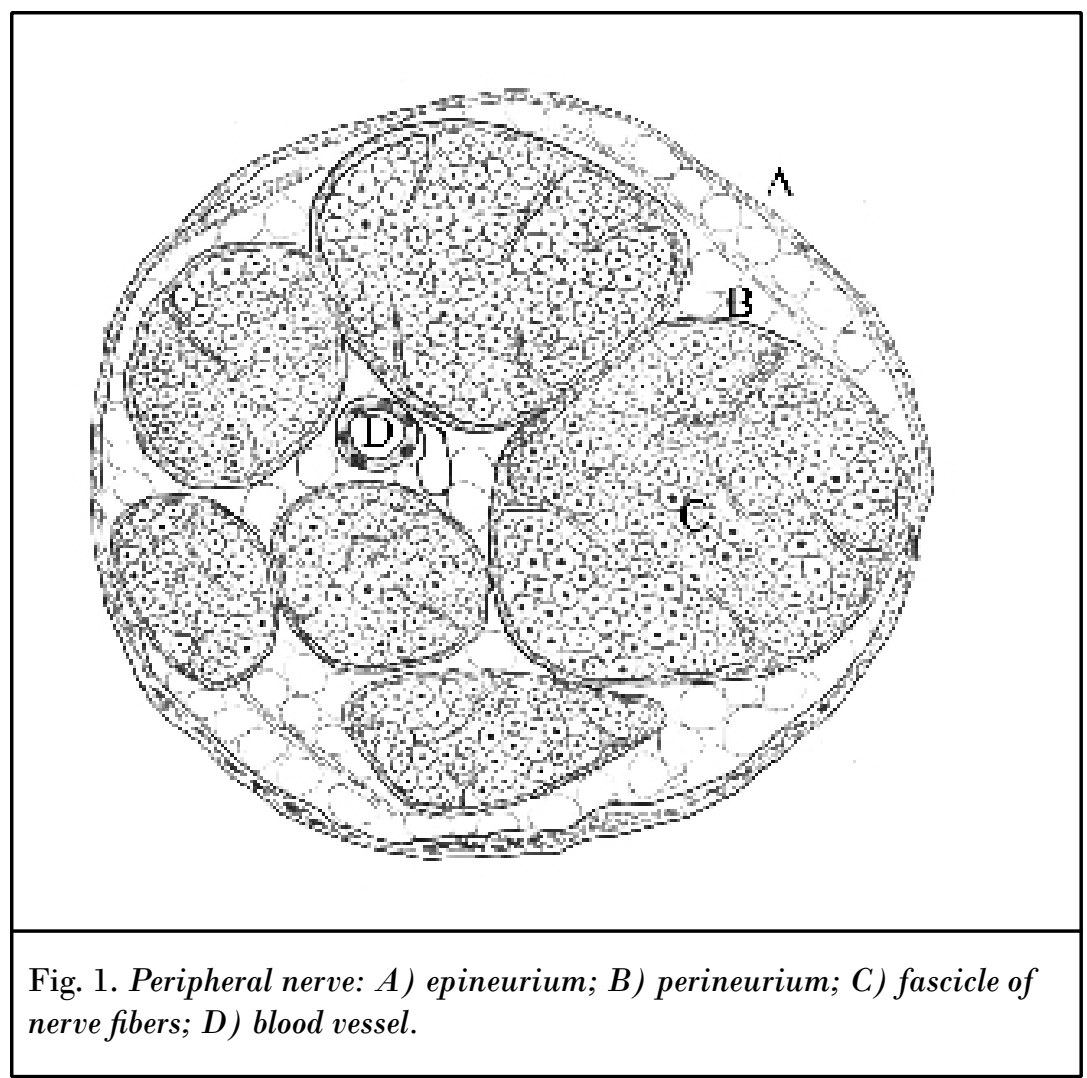

ume and concentration of local anesthetic required for successful regional anesthesia may differ significantly.

\section{Anatomy}

Peripheral nerves are generally mixed nerves containing afferent and efferent fibers of different size and structure, although some smaller peripheral nerves are purely sensory. All peripheral nerves are extensions of spinal nerves that exit the spinal column through the intervertebral foramina.

The foramen contains the dorsal root ganglia, spinal nerves, arteries, and veins. The dorsal root ganglion is a complex structure that contains several thousand cell bodies of afferent sensory axons.

Peripheral nerves often travel adjacent to blood vessels within neurovascular bundles, surrounded by layers formed by muscle fascia. The peripheral nerve itself is invested with epineurium that is loosely attached to adjacent nerve tissue (Fig. 1). The epineurium consists of dense irregular connective tissue surrounding groups of nerves and smaller blood vessels. A peripheral nerve contains a number of nerve fascicles, each enveloped by perineurium and separated from each other by additional fat and connective tissue. Each fascicle contains individual nerve fibers, connective tissue, and blood vessels. Individual axons are covered by glia cells that form the thin endoneurium. Nerve fibers of the autonomic nervous system travel to the periphery or innervate vascular structures within the peripheral nerve or nerve fascicle.

Myelinated motor and sensory nerve fibers are individually enveloped by layers of myelin, whereas small unmyelinated fibers such as nociceptive afferent $\mathrm{C}$ fibers are bundled and covered by a single Schwann cell sheath without covering layers of myelin.

Myelin is a bilayer lipid membrane formed by a Schwann cell that invests an individual myelinated nerve axon in as many as several hundred layers of myelin. The layers of myelin formed by a sinof Ranvier. The electrical impulse is transmitted in a saltatory fashion from node to node, with the myelin acting as insulation between the cell membrane and the extracellular space. Most of the sodium and potassium channels in myelinated nerves are present at these nodes. gle Schwann cell are interrupted by nodes

\section{Cell Physiology}

Like all other biologic membranes, the membrane of the nerve cell consists of a lipid bilayer with embedded cholesterol, carbohydrates, lipids, and proteins. The bilayer is formed by amphiphilic phospholipids with a polar hydrophilic head and a long hydrophopic hydrocarbon tail. The cell membrane is impermeable to ions and forms an electrical insulation between the inside and the outside of the nerve cell. The cell membrane is traversed by proteins with different molecular properties. These proteins act as selective ion channels permitting the different ions to cross the cell membrane. Several different subgroups of selective sodium, calcium, and potassium channels have been identified.

While all sodium channels have similar structures, there is variability in their distribution in different tissues and during different stages of development. The difference in sodium channel expression has an important effect on the different physiology and the effect of sodium channel blockers on different cells. Not only is there a difference of sodium channel expression in different tissue, but expression of sodium channels may vary among cells of the same tissue type. The sodium channel itself is a complex of glycosylated proteins, consisting of four -subunits and 1 and 2 subunits, which, in assembled form, traverse the bilayer cell membrane.

Triggered by depolarization of neighboring cell membrane, the sodium channel changes its conformation and opens for a few milliseconds. Depolarization changes the configuration of sodium channels to an open state, allowing a rapid influx of sodium ions through neighboring sodium channels in the area of the developing action potential.

During this open state sodium influx along the concentration gradient changes the local membrane potential and transmits the electrical impulse along the nerve.

Following depolarization, sodium channel are temporally refractory to additional depolarization impulses, and are in a non-conducting, inactivated closed state. During this time, the axon is in a repolarization phase, during which time the cell membrane is resistant to further depolarization. This resistance can be explained by the three conformational states of the sodium channel. The normal state 
of the sodium channel is the closed resting state, in which the sodium channel is impermeable to sodium. The opened state, during depolarization, is associated with increased inward sodium conductance. The inactivated state during which repolarization occurs, results from decreased sodium and increased potassium conductance that causes increased outward flux of potassium from the nerve cell into the extracellular space.

Sodium channel activation is required by the nerve to generate an action potential and to conduct a nerve impulse. By activating other neighboring voltagegated sodium channels the action potential spreads along the cell membrane in a self-propagating process. The short refractory period after depolarization, where the area of the cell membrane is inactivated, ensures a unidirectional impulse.

Depolarizations that do not recruit enough sodium channels are considered to be below the membrane excitability threshold and will not conduct an impulse. The velocity of impulse transmission along the axon depends on the diameter of unmyelinated nerves and whether or not the nerve axon is myelinated.

The concentration of ions in the nerve cell and the extracellular fluid differ markedly. The concentration of potassium ions is low in the extracellular space and high inside the axon; the concentration of sodium ions is high in the extracellular space and low within the cell. This gradient for sodium and potassium is maintained by an ion pump mechanism driven by $\mathrm{Na}+\mathrm{K}+$ ATPase, which is a membrane-bound protein located on the inside of the neural cell membrane. The process is energy-dependant, powered by ATP; three sodium ions are transported outward in exchange for two potassium ions transported inward.

At rest, the cell membrane is more permeable to potassium than to sodium ions, resulting in a net flow of potassium ions from inside the cell to the extracellular space along a concentration gradient. This leakage of potassium ions causes an electric potential of -60 mVolts to -90 mVolts across the cell membrane, with the inside of the membrane considered negative by definition. This electrical gradient is described by the Nernst equation.

Local anesthetics act primarily on voltage-gated sodium channels. By binding to the sodium channel the local anes- thetic prevents a conformational change of the channel and inhibits the fast sodium influx needed to initiate an action potential. Binding of local anesthetic molecules initially decreases the rate and strength of a nerve impulse and if sufficient, subsequently abolishes impulse transmission completely.

\section{Molecular Mechanisms of Action}

Most local anesthetics are basic, tertiary amine compounds that exist in solution either in charged (cationic) or uncharged (nonionic) form, depending on the pKa of the specific local anesthetic and the $\mathrm{pH}$ of the solution. The $\mathrm{pKa}$ of a compound describes the $\mathrm{pH}$ of the solution where half of the local anesthetic molecules exist in the charged and uncharged forms. In general, lowering the $\mathrm{pH}$ of a solution will increase the number of cationic molecules of local anesthetic, whereas increasing the $\mathrm{pH}$ will lower the number of charged molecules. If the $\mathrm{pH}$ is raised sufficiently, the anesthetic will precipitate out of solution, because the uncharged molecule has a low aqueous solubility.

Local anesthetics are weak bases with pKas ranging from 7.5 to $9.0(12,13)$.

Since they are relatively insoluble, local anesthetics are marketed as mildly acidic hydrochloride salts. In an acidic $\mathrm{pH}$ environment, most of the local anesthetic molecules are present in solution in their cationic, hydrophilic form. Indeed, when the $\mathrm{pH}$ of a solution is lower than the $\mathrm{pKa}$ of the local anesthetic, a larger fraction of molecules of anesthetic will be in the charged form.

Only the nonionic, lipid-soluble form of the local anesthetic can readily penetrate the different tissue layers and membranes. At a $\mathrm{pH}$ lower than the $\mathrm{pKa}$ of the local anesthetic, the amount of molecules penetrating biologic membranes depends greatly on the membrane surface charge (3). This explains why local anesthetics have a delayed onset of the nerve block and a patchy density, when the clinician attempts to anesthetize tissue surrounding a localized infection or abscess with a low $\mathrm{pH}$.

Both charged and uncharged local anesthetic molecules can block Na channels. But, for both groups of local anesthetics, esters and amides, the octanol: water buffer partition coefficient correlates with the degree of sodium channel blockade on small membrane patches, and therefore relative anesthetic poten- cy. Since the diffusion barriers in were removed, this indicates that the mechanism of action on peripheral nerve channels is mediated through lipophilic drug-channel interactions with the nonionic local anesthetic molecule (4).

As noted above, local anesthetics interrupt the transmission of nerve impulses by preventing the propagation of action potentials along the nerve cell membrane. At this time, the most widely accepted mechanism of action is local anesthetic binding to channel receptors on the inner portion of the membrane. The sodium channel undergoes a conformational change, which blocks the influx of sodium ions and prevents localized membrane depolarization and propagation of the action potential down the nerve. Other potential mechanisms include physical obstruction of the external opening of the sodium channel and intrachannel blockade. Because of the structural variety of substances with local anesthetic properties (e.g., calcium channel blockers, meperidine) it seems unlikely that sodium channel blockade is the sole effect of local anesthetic action. Indeed, local anesthetic not only block the inward sodium current, but also the outward potassium current through different potassium channels in peripheral nerves, dorsal root ganglion cells and in the dorsal horn (4-7). In addition, although most studies have focused on the effects of local anesthetic on sodium and potassium channels, some studies have demonstrated an effect on calcium currents $(8,9)$. Although the affinity of local anesthetics is lower for potassium and calcium channels than it is for sodium channels, block of potassium channels may contribute to the effect of local anesthetics, because potassium currents are responsible for neural repolarization and maintenance of the cell resting potential.

Sodium channels can be grouped based on their sensitivity to different toxins, such as tetrodotoxin (TTX). TTX is a toxin with high affinity for certain subgroups of sodium channels, causing virtually complete and selective blockage of that type of sodium channel. Although current evidence indicates that local anesthetics act primarily by stabilizing neural membrane by blockade of TTX-sensitive sodium channels, local anesthetics may also block TTX-resistant sodium channels (10). TTX-resistant sodium channels are found in the soma of small dorsal 
root ganglion neurons and may be upregulated after neural injury. In chronic pain states, blockade of TTX-resistant sodium channels on injured A-delta and C fibers may play an important role in blockade of painful nerve impulses (11-15).

During the early onset of regional anesthesia, impulse transmission along the nerve is not completely blocked. Instead, local anesthetics will decrease the frequency and the amplitude of the action potential and will alter the encoded information transported to the secondary neurons in the spinal cord. In rats, lidocaine and bupivacaine both decrease the amplitude of TTX-resistant action potentials in dorsal root ganglia small cells. The time to peak action potential and duration of TTX-resistant action potentials are prolonged (16).

Local anesthetics block sensory and motor fibers to a different degree. The concentrations needed to reduce the amplitude of TTX-resistant action potentials, compared to TTX-sensitive action potentials is lower for bupivacaine than for lidocaine. This may explain why bupivacaine, at a given concentration, will block sensory fibers, but not motor fibers, to the same extent as lidocaine (15). Studies have shown that concentrations of lidocaine necessary to block $\mathrm{C}$ fiber nociceptors is approximately $1.0 \mathrm{mM}$. On the other hand, $0.5 \mathrm{mM}$ will block A-delta-fiber nociceptors and A-beta-mechanoreceptors $(17,18)$. The different structure of sodium channels, their distribution throughout the nervous system, and the affinity of different local anesthetics for the various channels, influences the extent of sensory and motor blockade and accounts for the potential toxicity of local anesthetics.

\section{Mass Effect of Local Anesthetic}

The key to success for a nerve block is to deposit the local anesthetic as close to the nerve as possible. Depending on the nerve block and where the local anesthetic is injected, the anesthetic molecules need to cross a number of structures to reach the binding site at the sodium-channel of the neuron.

For spinal anesthesia, the local anesthetic acts on nerve rootlets, which are free-floating in the cerebrospinal fluid. The only structures to be penetrated by the local anesthetic are the different layers of peri-, epi-, and endoneurium. Thus, local anesthetic is injected in close proximity to neural tissue, penetrates the neu- ral layers quickly, and the onset of blockade is rapid.

In peripheral nerve blocks the local anesthetic is deposited in fat tissue, muscle, or potential spaces between different anatomical structures. The local anesthetic must spread along these different anatomical structures. Blockade is dependent on spread, which is a mass effect and is proportional to the volume of anesthetic injected. To reach the nerve itself, anesthetic molecules must penetrate by diffusion multiple connective tissue layers, fat, per-, epi-, and endoneurium to reach sodium channels that reside inside the cell membrane of the axon.

The different techniques used to identify the proper injection site adjacent to a nerve influence the success rate, the speed of onset, and the quality of the block. Indirect techniques, which rely solely on anatomic landmarks to identify nerve location, without electing paresthesias or using nerve stimulation, may place the injected local anesthetic at a significant distance from the target peripheral nerve. The same holds true for identifying the neurovascular bundle by feeling a "fascial click," where the needle is presumed to penetrate the fascia surrounding the neurovascular bundle. In clinical use, some of these potential pitfalls are overcome by a sufficiently large volume of local anesthetic. However, larger volumes (and resulting larger doses of drug) increase the risk of systemic side effects.

Recent studies have identified the actual distribution of local anesthetic with the help of MRI and CT imaging during the performance of different regional blocks. MRI-imaging, obtained after injection of $50 \mathrm{ml}$ of local anesthetic through an axillary catheter, showed an average longitudinal spread of $92 \mathrm{~mm}$ and a large cross-sectional spread after 30 minutes (19). These imaging studies do not support the concept, as postulated in earlier publications, of a tubular brachial plexus sheath surrounding the neurovascular bundle, containing the injected local anesthetic. Instead, the local anesthetic seems to spread along anatomical structures over time, with a wide cross-sectional distribution $(20,21)$.

MRI imaging used to identify the distribution of local anesthetic in threein-one blocks showed similar results. The local anesthetic spreads laterally, caudally, and to a small extent medially, to the site of injection. This is consistent with ana- tomic cadaver studies, which were not able to identify a sheath extending from the lumbar plexus to the femoral nerve. These findings contradict previous teaching that local anesthetics spread cephalad from the site of an inguinal injection to reach the lumbar plexus $(22,23)$.

With respect to these studies, the large volumes suggested for regional blocks, such as a three-in-one block or a brachial plexus block, are not required to spread alongside the nerve and cover a large distance or even reach the plexus, but rather to allow sufficient spread along anatomical structures to reach more distant and less accessible nerves from the injection site, with the minimal blocking concentration of the local anesthetic (24). That same study showed that local anesthetic concentration, rather than dose, is the parameter for a successful nerve block. With the same dose of lidocaine, a higher concentration but smaller volume resulted in a more intense and longer lasting block (24).

Local anesthetic injected into the tissue surrounding the nerve will spread in all directions along anatomical planes. The success of the block depends on the volume of local anesthetic and concentration that bathes the nerve. The use of ultrasound to identify anatomical structures including large peripheral nerves, and the use of nerve stimulation to pinpoint the exact location of a nerve and assure close proximity of the injected local anesthetic to the nerve, will ensure blocks of higher quality and reduce potential toxic effects by reducing the volume and the amount of local anesthetic needed.

On the other hand, large volumes and higher concentrations used for a peripheral nerve block compared to spinal anesthesia will prolong the time of vascular uptake, redistribution, and metabolism of local anesthetic. The same layers and tissue preventing a fast onset of regional anesthesia are responsible for prolonging the duration of the regional blockade.

\section{Onset of Neural Blockade}

There is a predictable variability in loss of different nerve functions, but in most cases the loss of sensation and motor function overlaps. Usually, the first neural function to be lost is the sensation of pain, followed by the loss of sensations of cold, warmth, touch, pressure, and finally motor function. This phenomenon 
was noted in the early days of regional anesthesia and given the name differential nerve block. Initially, it was believed that the different diameter of nerve fibers was responsible for the differential block and that small fibers would be blocked before larger ones.

The onset of regional anesthesia and the loss of function is influenced by the following: proximity of the injection site to the nerve; the intervening tissue between the injection site and the nerve; the volume, concentration and pharmacokinetic properties of local anesthetics; the electro-physiologic properties of the nerve; the $\mathrm{pH}$ of the surrounding tissue; and the anatomic properties and location of the individual nerve fibers within the nerve bundle. In this regard, nerve fibers closer to the periphery will be blocked earlier than will nerve fibers in the core of a nerve bundle. The size of the nerve also plays a role; in theory small diameter nerves are more easily blocked than are large diameter nerves. The internodal distance, which is longer in larger nerves, may also play an important role in the more rapid blockade of sensory fibers than motor fibers. Several sequential nodes of Ranvier must be blocked in succession to achieve a solid block. Since the nodes of Ranvier are closer together in myelinated small diameter fibers than in large fibers, more nodes could be blocked at a given length. The different time of onset of the regional block may also be influenced by the encoding of neural impulses by patterns. Sensory qualities are not transmitted by single action potentials along a nerve, but as different patterns.

As mentioned earlier, small doses of local anesthetic change the frequency and strength of a neural impulse before the impulse is completely abolished. The encoding of different neural functions may be more susceptive to lower concentrations of local anesthetic in the early stage of a regional block. This would explain the different time of loss of neural function in nerves that are otherwise anatomically identical.

\section{Duration and Quality of Neural BLOCKADE}

Sodium bicarbonate is often added to the local anesthetic solution by clinicians to increase the $\mathrm{pH}$ and decrease the pain of injection of acidic local solutions. In theory, the addition of sodium bicarbonate will also convert more local anes- thetic molecules into the nonionic form, thereby increasing the rate of penetration of local anesthetic through the different layers of tissue and shortening the onset of anesthesia. However, the literature concerning the clinical effect of adding sodium bicarbonate to local anesthetic is controversial.

Due to the poor water solubility of local anesthetic, the $\mathrm{pH}$ of the solution can be increased with sodium bicarbonate to only a small degree without causing precipitation of the drug. Some studies showed a more rapid onset and a better quality of epidural anesthesia with the addition of $2 \mathrm{ml}$ sodium bicarbonate compared to epidural anesthesia with plain 2\% lidocaine without Epinephrine (25), while other studies showed no difference in onset time but postulated a denser block with addition of sodium bicarbonate (26).

In a rat model of a sciatic nerve block, the addition of sodium bicarbonate to $1 \%$ lidocaine without epinephrine decreased the degree and duration of the regional block, but did not affect the time of onset. In the same study, the addition of sodium bicarbonate to lidocaine $1 \%$ with epinephrine did shorten the onset time without affecting degree and duration (27).

The above notwithstanding, the pain associated with lidocaine injection, particularly into the dermis and deeper tissues (prior to insertion of a larger needle for interventional pain procedures) is clinically significantly less with buffered lidocaine. Unfortunately, bupivacaine precipitates out of solution with a slight increase in $\mathrm{pH}$.

The offset of regional anesthesia is caused by the systemic uptake of local anesthetics and a decreasing concentration at the nerve membrane. Lipophilic local anesthetics, such as bupivacaine will remain for a longer time in the tissue surrounding the nerve than will a less lipophilic agent such as lidocaine.

The addition of vasoconstrictors to the local anesthetic solution counteracts the vasodilating properties of some local anesthetics and prolongs the duration of blockade by delaying vascular uptake of local anesthetic. The effect can prolong the block by as much as $50 \%$, depending on the particular local anesthetic and its vasodilating properties.

For sciatic block in a rat study (28), the addition of epinephrine $(1: 100,000)$ to
$0.5 \%$ lidocaine increased the intensity and duration of anesthesia as well as the percentage of animals with complete block.

\section{ConCLUSIONS}

Peripheral nerves transporting afferent signals to the spinal cord are comprised of nerve fascicle bundles surrounded by perineurium, whereas individual axons are invested with endoneurium, and the peripheral nerve itself is covered by epineurium. Neural blockade occurs either at any site of a peripheral nerve, from the periphery to the spinal nerve or as a neuroaxial block, affecting the nerve rootlets proximal of the dorsal root ganglia. The historical idea of a neurovascular bundle encased by a common sheath could not be confirmed by modern imaging studies.

Local anesthetics cause reversible blockade of nerve impulse generation and propagation. Blockade occurs by inhibition of sodium and potassium channel function, with additional affects on calcium channel activity.

Most local anesthetics are tertiary amines that exist in charged and uncharged form, depending on $\mathrm{pH}$, and are weak bases. Local anesthetics are marketed as mildly acidic salts (e.g., of hydrochloric acid). Acidic environments delay onset of local anesthetic blockade by an increase of charged local anesthetic molecules, with restricted permeability through biologic membranes. Nevertheless, cationic local anesthetic molecules seems to have a higher affinity to sodium channels than nonionic molecules. Neural blockade is a function of anesthetic concentration and volume. The mechanisms explaining the phenomenon of differential nerve blockade (loss of pain and temperature sensation before motor function) are complex and are still the subject of discussion.

Alkalinizing the anesthetic solution prior to use may reduce the pain with injection and reduce time to onset of blockade with lidocaine. Addition of epinephrine may increase duration blockade, counteract the vasodilating properties of the local anesthetic, and slow vascular uptake of local anesthetic. While some neural blocks depend on large volumes for adequate spread of local anesthetic solution to achieve the desired blockade, the risks and side effects also increase. As with most endeavors in clinical medicine, the quality of neural blockade is operator-depen- 
dent, and requires knowledge of the clinical characteristics of the local anesthetic, an accurate understanding of the relevant anatomy, and excellent technical skills. New pharmacological discoveries, new anatomical approaches for regional techniques based on modern imaging studies, new regional needle designs, and the use of guiding techniques like CT, ultrasound, and nerve stimulation will improve the success rate of neural blocks and increase patient and physician satisfaction.

Author AfFILATION:

Andreas Grabinsky, MD

Assistant Professor of Anesthesiology

Metro Health Medical Center and

Case Western Reserve University School

of Medicine

Cleveland, $\mathrm{OH} 44106$

E-mail: grabi@mac.com

\section{REFERENCES}

1. Galbis-Reig D. Sigmund Freud and Carl Koller: the controversy surrounding the discovery of local anesthesia. International Congress, Series 2002; 1242:571-575.

2. Boswell MV, lacono RP, Guthkelch AN. Sites of action of subarachnoid lidocaine and tetracaine: observations with evoked potential monitoring during spinal cord stimulator implantation. Reg Anesth 1992; 17:37-42.

3. Ohki S, Ohshima H. Distribution of local anesthetic in lipid membranes. Colloids Surf B Biointerfaces 1996; 5:281-305.

4. Brau ME, Vogel W, Hempelmann G. Fundamental properties of local anesthetics: half-maximal blocking concentrations for tonic block of $\mathrm{Na}+$ and $\mathrm{K}+$ channels in peripheral nerve. Anesth Analg 1998; 87: 885-889.

5. Kindler CH,Yost CS, Gray AT. Local anesthetic inhibition of baseline potassium channels with two pore domains in tandem. Anesthesiology 1999; 90:1092-1102.

6. Komai H, McDowell TS. Local anesthetic inhibition of voltage-activated potassium currents in rat dorsal root ganglion neurons. Anesthesiology 2001; 94:1089-1095.

7. Olschewski A, Hempelmann G, Vogel W, Safronov BV. Blockade of $\mathrm{Na}+$ and $\mathrm{K}+$ currents by local anesthetics in the dorsal horn neurons of the spinal cord. Anesthesiology 1998; 88:172-179.

8. Hollmann MW, Durieux ME. Local anesthetics and the inflammatory response: a new therapeutic indication? Anesthesiology 2000; 93:858-875.

9. $\mathrm{Xu} \mathrm{F,} \mathrm{Garavito-Aguilar} \mathrm{Z,} \mathrm{Recio-Pinto}$ E, Zhang J, J Blanck TJ. Local anesthetics modulate neural calcium signaling through multiple sites of action. Anesthesiology 2003; 98:1139-1146.

10. Roy ML, Narahashi T. Differential properties of tetrodotoxin-sensitive and tetrodotoxin-resistant sodium channels in rat dorsal root ganglion neurons. J Neurosci 1992; 12:2104-2111.

11. Ritter AM, Mendell LM. Somal membrane properties of physiologically identified sensory neurons in the rat: effects of nerve growth factor. I Neurophysiol 1992; 68:2033-2041.

12. Jeftinija $\mathrm{S}$. The role of tetrodotoxin-resistant sodium channels of small primary afferent fibers. Brain Res 1994; 639125-134.

13. Quasthoff S, Grosskreutz J, Schroder JM, Schneider U, Grafe P. Calcium potentials and tetrodotoxin-resistant sodium potentials in unmyelinated C fibres of biopsied human sural nerve. Neuroscience 1995; 69:955-965.

14. Rabert DK, Koch BD, Ilnicka M, Obernolte RA, Naylor SL, Herman RC, Eglen RM, Hunter JC, Sangameswaran L. A tetrodotoxin-resistant voltage-gated sodium channel from human dorsal root ganglia, hPN3/ SCN10A. Pain 1998; 78:107-114.

15. Scholz A, Kuboyama N, Hempelmann G, Vogel W. Complex blockade of TTX-resistant Na1 currents by lidocaine and bupivacaine reduce firing frequency in DRG neurons. J Neurophysiol 1998; 79:1746-1754.

16. Scholz A, Vogel W. Tetrodotoxin-resistant action potentials in dorsal root ganglion neurons are blocked by local anesthetic. Pain 2000; 89:47-52.

17. Huang JH, Thalhammer JG, Raymond SA, Strichartz GR: Susceptibility to lidocaine of impulses in different somatosensory afferent fibers of rat sciatic nerve. J Pharma- col Exp Ther 1997; 292:802-811.

18. Gokin AP, Philip B, Strichartz GR. Preferential block of small myelinated sensory and motor fibers by lidocaine: In vivo electrophysiology in the rat sciatic nerve. Anesthesiology 2001; 95:1441-1454.

19. Klaastad O, Smedby O, Thompson GE, Tillung T, Hol PK, Rotnes JS, Brodel P, Breivik $\mathrm{H}$, Hetland KR, Fosse ET. Distribution of local anesthetic in axillary brachial plexus block: A clinical and magnetic resonance imaging study. Anesthesiology 2002; 96: 1315-1324.

20. De Jong RH. Axillary block of the brachial plexus. Anesthesiology 1961; 22:215-225.

21. Thompson GE, Rorie DK. Functional anatomy of the brachial plexus sheaths. Anesthesiology 1983; 59:117-122.

22. Ritter JW. Femoral nerve "sheath" for in guinal paravascular lumbar plexus block is not found in human cadavers. J Clin Anesth 1995; 7:470-473.

23. Marhofer P, Nasel C, Sitzwohl C, Kapral S. Magnetic resonance imaging of the distribution of local anesthetic during the threein-one block. Anesth Analg 2000; 90:119124.

24. Nakamura T, Popitz-Bergez F, Birknes J, Strichartz GR. The critical role of concentration for lidocaine block of peripheral nerve in vivo: Studies of function and drug uptake in the rat. Anesthesiology 2003; 99:1189-1197.

25. Curatolo M, Petersen-Felix S, ArendtNielsen L, Lauber R, Hogstrom H, Scaramozzino P, Luginbuhl M, Sieber TJ, Zbinden AM: Adding sodium bicarbonate to lidocaine enhances the depth of epidural blockade. Anesth Analg 1998; 86:341347.

26. Gosteli P, Van Gessel E, Gamulin Z. Effects of $\mathrm{pH}$ adjustment and carbonation of lidocaine during epidural anesthesia for foot and ankle surgery. Anesth Analg 1995; 81: 104-109.

27. Sinnott CJ, Garfield JM, Thalhammer JG, Strichartz GR. Addition of sodium bicarbonate to lidocaine decreases the duration of peripheral nerve block in the rat. Anesthesiology 2000; 93:1045-1052.

28. Sinnott CJ, Cogswell III LP, Johnson A, Strichartz GR. On the mechanism by which epinephrine potentiates lidocaine's peripheral nerve block. Anesthesiology 2003; 98:181-188. 